Child Neuropsychology Vol. 12(2):125-140 (2006)

ISSN: (print 0929-7049)(online 1744-4136)

doi:10.1080/09297040500499081

This is a peer reviewed pre-print version of the following article: Reaction Time Distribution Analysis of

Neuropsychological Performance in an ADHD Sample, which has been published in final form at:

http://www.psypress.com

http://www.tandf.co.uk/journals/titles/09297049.asp

http://www.tandfonline.com/doi/pdf/10.1080/09297040500499081

(C) Taylor \& Francis Group, LLC

\title{
Reaction Time Distribution Analysis of Neuropsychological Performance in an ADHD Sample
}

\begin{abstract}
Aaron S. Hervey, Brigham and Women's Hospital, and Harvard Medical School, Jeffery N. Epstein, Duke University Medical Center, John F. Curry, Duke University Medical Center, and Duke University Department of Psychology: Social Health Sciences, Simon Tonev, Duke University Medical Center, L. Eugene Arnold, Ohio State University, C. Keith Conners, Duke University Medical Center, Stephen P. Hinshaw, University of California at Berkeley, James M. Swanson, University of California at Irvine, and Lily Hechtman Montreal Children's Hospital
\end{abstract}

\begin{abstract}
Differences in reaction time (RT) variability have been documented between children with and without Attention Deficit Hyperactivity Disorder (ADHD). Most previous research has utilized estimates of normal distributions to examine variability. Using a nontraditional approach, the present study evaluated RT distributions on the Conners' Continuous Performance Test in children and adolescents from the Multimodal Treatment Study of ADHD sample compared to a matched sample of normal controls ( $\mathrm{n}=65$ pairs). The ex-Gaussian curve was used to model RT and RT variability. Children with ADHD demonstrated faster RT associated with the normal portion of the curve and a greater proportion of abnormally slow responses associated with the exponential portion of the curve. These results contradict previous interpretation that children with ADHD have slower than normal responding and demonstrate why slower RT is found when estimates of variability assume normal Gaussian distributions. Further, results of this study suggest that the greater number of abnormally long RTs of children with ADHD reflect attentional lapses on some but not all trials.
\end{abstract}

\section{Introduction}

For several decades, researchers have attempted to understand the cognitive processes impaired in patients with Attention Deficit Hyperactivity Disorder (ADHD). While behavioral features of the disorder such as hyperactivity and impulsivity are more easily observed than cognitive components of the disorder, the former often cause more serious impairment, especially in academic activities. Numerous neuropsychological studies have been conducted with children and adults with ADHD attempting to define core cognitive deficits (see Hervey et al., 2004 and Pennington \& Ozonoff, 1996 for reviews). Over the years, core deficits in behavioral inhibition, working memory, and various aspects of attention have been noted and assimilated into various theoretical frameworks (e.g., Barkley 1997b; Douglas 1999; Denney \& Rapport, 2001; Sergeant \& van der Meere, 1990).

Within the attention domain, one of the areas of noted neuropsychological test performance impairment in children with ADHD is performance consistency. This is most often measured by examining patterns of reaction time (RT) on various neuropsychological tasks. Several authors have argued the importance of performance variability in ADHD (Douglas, 
1999) and the importance of RT as a reflection of attention. Van der Molen (1996) refers to RT variability as the "dynamometer of attention," with high variability indicative of fluctuations or lapses in attention.

Empirical investigations have found that children with ADHD frequently demonstrate impaired performance with slower mean RT compared to nonclinical controls (Teicher, Ito, Glod, \& Barber, 1996; Klorman, Salzman, Pass, Borgstedt, \& Dainer, 1979). However, more common is the finding that children with ADHD demonstrate greater RT variability than nonclinical control counterparts on a variety of speeded RT tasks regardless of sensory domain (Teicher, Ito, Glod, \& Barber, 1996; Douglas, 1999; Kuntsi, Oosterlaan, \& Stevenson, 2001; Pennington, Groisser, \& Welsh, 1993; Sergeant \& van der Meere, 1990; Cohen \& Douglas, 1972; Zahn, Kruesi, \& Rapoport, 1991).

Most neuropsychological investigations have utilized summary statistics that represent global performance (i.e., RT, RT standard deviation, etc.) as outcome measures. Although these measures provide performance information, this information is limited in that it generally represents a summation of performance from a large pool of data, usually collected across many trials, rather than a more detailed analysis of the composition of the data. As Castellanos and Tannock (2002) suggest, the intraindividual variability or moment-by-moment process of task performance in which individuals with ADHD demonstrate difficulty is not reflected by these overall statistics. It is not the case that children with ADHD cannot pay attention at all. Rather, these children demonstrate attention lapses more frequently than do nonclinical controls. What is unique to patients with ADHD is the inconsistency with which these behaviors are exhibited. Several authors (Castellanos and Tannock, 2002; Kuntsi, Oosterlaan, \& Stevenson, 2001; Kuntsi $\&$ Stevenson, 2001) have argued that performance variability is the essence of ADHD and emphasize the roles that temporal and contextual variables play in this inconsistency. Recent neuroimaging findings also confirm that response variability is related to a distributed brain network including brain regions (i.e., frontal lobes) consistently implicated in the pathophysiology of ADHD (see Giedd et al., 2001 for review).

More recently, this performance inconsistency has been investigated as the basis for irregular performance based on both theoretically and empirically driven models. For example, utilizing a data distribution analytical technique, the activation-suppression hypothesis (Ridderinkhof, 2002) suggests that there are two pathways through which RT responses are made, direct response activation and a deliberate response decision process, the latter of which necessitates suppression of activation in order to occur. Based on differential RT performance associated with distraction, this theory has been used to implicate response inhibition as a fundamental neurocognitive deficit in ADHD (Ridderinkhof, Scheres, Oosterlaan, \& Sergeant, in press).

Detailed RT analysis has also been used to implicate impaired attention in ADHD. Leth-Steensen, Elbaz, and Douglas (2000) attempted to conduct a more fine-grained, detailed analysis of response patterns of children with ADHD utilizing a simple and well-conceived RT data analysis approach. Using a choice RT task (Iaboni, Douglas, \& Ditto, 1997), RTs from each trial were compiled to generate a RT distribution to model an ex-Gaussian density curve. Briefly, the ex-Gaussian curve, originally developed by Burbeck and Luce (1982), represents the additive combinations of the independent Gaussian (normal) distribution and exponential random variables, the latter portion of which makes up the positive skew of the distribution curve. The ex-Gaussian distribution has three parameters: $\mu(\mathrm{mu})$, the mean of the normal component, $\sigma$ (sigma), the standard deviation of the normal component, and $\tau$ (tau), a value describing both the 
mean and the standard deviation of the exponential component and representing the key area of interest in their study. According to Leth-Steensen, Elbaz, and Douglas, tau theoretically represents the most sensitive measure of RT in children with ADHD. They proposed and subsequently demonstrated that children with ADHD perform similarly to nonclinical controls in terms of their RT and RT variability using the normal curve, but they were highly discrepant from normal controls on the ex-Gaussian tau measure, but not mu or sigma (Leth-Steensen, Elbaz, \& Douglas, 2000). Greater tau indicates more frequent excessively long RTs, which is represented as positive skew in the ex-Gaussian distribution. This is different than a general slowing of RT. While a general slowing might indicate a variety of unspecified difficulties with basic cognitive processes, periodic excessively long RT has been argued to be more likely a consequence of poor attention. These trials have been hypothesized to be the occasions where children with ADHD demonstrate lapses in attention (Douglas, 1999; Leth-Steensen, Elbaz, \& Douglas, 2000).

While Leth-Steensen et al.'s results are interesting, they are based on a small total sample (ADHD $n=17$ ), they have not been replicated, and they have not been generalized to tasks other than a forced-choice RT test. Moreover, the task used in the Leth-Steensen et al. study is not a task that has been used frequently either clinically or for ADHD research studies.

Go/No-Go tests are frequently used measures to assess children with ADHD. The Conners' Continuous Performance Test (CPT) is a Go/No-Go test that includes a high number of trials measuring RT thus permitting more in-depth evaluation of RT data. The present study evaluated RT data using the Conners' CPT in children with ADHD. It was predicted that this investigation would replicate the findings of Leth-Steensen, Elbaz, and Douglas. A similar pattern of findings would serve as evidence in support of the hypothesis that children with ADHD demonstrate greater variability in RT as a result of periodic exceptionally long RT trials, rather then as a result of general slowness to respond. In addition, greater values of tau for children with ADHD compared to nonclinical controls, combined with similar values of mu and sigma, may eventually prove to be a more specific performance pattern for identifying children with ADHD.

\section{Methods}

\section{Subjects}

Data for this study were collected as part of the 24-month follow-up evaluation of the six-cite Multimodal Treatment Study of children with ADHD (MTA). During baseline assessment for the MTA study, all included children $(n=579)$ received a diagnosis of ADHD, Combined Type (American Physical Association, 1994). This diagnosis was determined primarily using the Diagnostic Interview Schedule for Children, Parent Report (DISC-P 3.0; Shaffer et al., 1996), supplemented with up to two symptoms identified by children's teachers using the SNAP-IV (DSM-IV ADHD/ODD Scale; Swanson, 1992) for cases falling just below the DISC diagnostic threshold. Once a diagnosis was confirmed, children were randomly assigned to one of four treatment groups: Medication Only, Psychosocial Treatment Only, Combined Treatment, or Community Control. The treatment phase lasted for 14 months. A more detailed description of the rationale, design, and results for the MTA study are presented elsewhere (Arnold, Abikoff, Cantwell, Conners, Elliott, Greenhill, Hechtman, et al. 1997; MTA Cooperative Group, 1999).

At the time of the 14-month assessment, families were no longer required to adhere to their randomized treatment and were allowed to seek any form of treatment for their child. At the 24-month assessment or 10 months after the end of treatment, all children were thoroughly assessed 
again using a comprehensive battery of measures. Across the four data collection sites that participated in this CPT study (baseline $n=387$ ), the retention rate of children who had completed DISC-P data at 24-months was $92 \%(n=356)$. Of these children, 319 had Conners' Continuous Performance Test data. Further, only 151 of these children met diagnostic criteria for ADHD at the 24-month assessment according to the DISC-P. Finally, in order to examine ADHD-related deficits in the absence of medication, only children with ADHD who were not taking medication on the day of the CPT assessment were utilized for this study (final $n=65$ ). Parents were requested to medicate their children on the day of testing if the child was medicated regularly and to refrain from medication if the child was typically unmedicated. These children comprised the ADHD sample for the present study. A nonclinical control group for the present study was derived from the Local Normative Comparison Group (LNCG) acquired at the time of the 24-month MTA assessment. LNCG children were living in the same communities and attending the same schools as the MTA children. These children ( $n=194$ across the four MTA sites) were identified from school registries to match the MTA sample in terms of grade, sex, and ethnicity and then randomly chosen from among those parents who volunteered to participate. For the purposes of this CPT study, those children in the LNCG group who met criteria for any subtype of ADHD based on DISC parent report $(n=20)$ were dropped from the study to avoid confounding the two groups.

A matching procedure was utilized to select one LNCG child for each ADHD child $(n=$ 65). LNCG participants were matched on four variables prioritized as follows: sex, ethnicity, age, and collection site. The matching criterion for age was within 6 months. A matched sample of 65 LNCG children was selected using these criteria. Demographic data for the matched ADHD and LNCG groups, including gender, ethnicity, age, and comorbid disorders are presented in Table 1.

\section{Measures}

\begin{tabular}{|c|c|c|c|}
\hline & $\operatorname{ADHD}(\mathrm{n}=$ & & Control $(n=65)$ \\
\hline Percentage male & 77 & 77 & \\
\hline \multicolumn{4}{|l|}{ Ethnicitv- } \\
\hline Caucasian & 37 & 41 & \\
\hline African American & 20 & 14 & \\
\hline Hispanic (non-Black) & 2 & 5 & \\
\hline Asian & 1 & 1 & \\
\hline Mixed & 5 & 4 & \\
\hline Age in years & $10.7(.84)$ & & $10.6(.93)$ \\
\hline \multicolumn{4}{|l|}{ Number of each ADHD Subtype } \\
\hline Inattentive & 26 & 0 & \\
\hline Hyperactive/Impulsive & 12 & 0 & \\
\hline Combined & 27 & 0 & \\
\hline \multicolumn{4}{|c|}{ Percentage with specified psvchological } \\
\hline Oppositional Defiant Disorder & 14 & 1 & \\
\hline Conduct Disorder & 6 & 0 & \\
\hline Any Anxiety Disorder & 27 & 9 & \\
\hline Any Elimination Disorder & 12 & 3 & \\
\hline Tic Disorder & 8 & 0 & \\
\hline Any Mood Disorder & 0 & 0 & \\
\hline
\end{tabular}

Note: Any Anxiety Disorders includes: Simple Phobia, Social Phobia, Agoraphobia, Panic Disorder, Overanxious Disorder, Generalized Anxiety Disorder, Obsessive-Compulsive Disorder, \& Separation Anxiety Disorder; Any Mood Disorder includes Major Depression, Dysthymia, Mania, \& Hypomania; Any Elimination Disorder includes enuresis (primary or secondary) and encopresis.

Table 1 Demographic Characteristics for ADHD and Control Group 


\section{Conners Continuous Performance Test.}

The Conners Continuous Performance Test (CPT; Conners, 1994) was completed on an IBM-compatible desktop computer in a quiet setting with minimizing distractions. Three hundred sixty (360) total letters appeared on the computer screen, one at a time, each for approximately 250 milliseconds. The 360 trials were presented in the standard format of 18 blocks of 20 trials each. The blocks differed only in the interstimulus intervals (ISI) between letter presentations, and lasted 1-, 2-, or 4-seconds. Interstimulus intervals were randomized between blocks so that all three ISI conditions would occur every three blocks. Transition from one block to the next was unannounced and occurred without delay. The CPT normally provides summary measures describing RT, including mean RT and RT standard error, the latter of which is identical to standard deviation, only conceptualizing an individual's RTs as a sampling distribution, rather than a population distribution (Howell, 1989; Keppel, 1991).

Participants were taking the CPT for the first time. They were instructed to press the spacebar when any letter except the letter " $X$ " appeared on the screen. The percentage of trials when letters other than " $X$ " appeared, was $90 \%$ across all ISI blocks. Reaction time was measured from the point at which any letter other than " $X$ " appeared on the screen until the spacebar was depressed. This is considered a Go trial. Only successful Go trials, or trials where the participant correctly pressed the spacebar when presented with a target stimulus were included for data analysis. No-Go trials occurred when an " $\mathrm{X}$ " was presented. Two types of errors were recorded. Errors of omission occured when the participant failed to respond to a target stimulus. Errors of commission occurred when the participant responded to a nontarget stimulus (i.e., "X"). The total Conners' CPT task takes approximately 14 minutes to complete.

Diagnostic Interview Schedule for Children-Parent Report (version 3.0).

Categorical DSM-IV diagnoses were made using the Diagnostic Interview Schedule for Children-Parent Version (DISC-P 3.0; Shaffer et al., 1996). The DISC is a structured diagnostic interview instrument designed for use in epidemiological and clinical studies by lay interviewers. It contains algorithms to generate diagnoses, based on rules similar to those published in the American Psychiatric Association's Diagnostic and Statistical Manual, Fourth Edition (American Psychiatric Association's, 1994).

\section{Procedure}

The MTA children and their parents participated in informed consent during the baseline visit of the MTA study. The LNCG participants and their parents were consented during the 24-month MTA follow-up, which served as the baseline visit for the LNCG group. Children in both groups were administered the CPT as part of a more comprehensive assessment lasting approximately 5 hours. The CPT was administered to the children as the second measure of a fixed assessment battery. Trained interviewers administered the DISC-P to parents and the results of this administration were scored using computerized algorithms.

\section{Data Processing}

RTSYS 1.0 (Heathcote, 1996) is a DOS based statistical program used for the analysis of RT data. The RTSYS program uses a theoretical distribution comprised of the combination of a Gaussian distribution in the initial or left side of the distribution curve, and an exponential distribution on the latter or right side of the distribution curve as a model of reaction time distribution. This type of distribution has been shown to create a better fit than traditional 
Gaussian distributions for RT (Luce, 1986). Specifically, it can more easily account for the positively skewed distribution commonly seen in reaction time data. This theoretical distribution, the ex-Gaussian, represents the sum of the independent Gaussian (normal) distribution and exponential random variables that comprise the exponential distribution and resulting positive skew of the curve. The ex-Gaussian distribution has three parameters: mu, the mean of the normal component, sigma, the standard deviation of the normal component, and tau, a single value that describes both the mean and the standard deviation of the exponential component. The tau component characterizes the skewness of the distribution. The parameters of mu and tau share a linear additive relationship such that $\mathrm{mu}+$ tau equals the overall mean of the distribution. Likewise, $S D$ equals the square root of sigma squared plus tau squared.

The RTSYS program uses each RT response recorded during the CPT administration for each participant to calculate $\mu, \sigma$, and $\tau$ for each individual. Calculations are estimated using a probability density function, a mathematical equation predicting the likelihood that a response will occur at any given time increment underneath a distribution curve. Parameter values for the probability density function are selected for each participant using maximum likelihood estimation, that is, the parameter values that are most likely given the data set. This estimation is achieved using an algorithmic search process known as Simplex.

The fastest RT data were trimmed using an absolute cutoff. Ulrich and Miller (1994) recommend using absolute censoring for rejecting fast RTs, which occur through anticipation. Luce (1986) has demonstrated that the nondecision portion of simple reaction time is at least 100 ms. In light of his recommendation, the present study used an absolute cutoff of $100 \mathrm{~ms}$. Any responses that occurred less than $100 \mathrm{~ms}$ following presentation of a stimulus were omitted from analysis, including any late responses from a preceding trial that registered within the first $100 \mathrm{~ms}$ of the next trial. On average, $5.9 \%$ of the trials of children with ADHD were censored, compared to $3.2 \%$ of the trials for the LNCG group (see Table 2). 


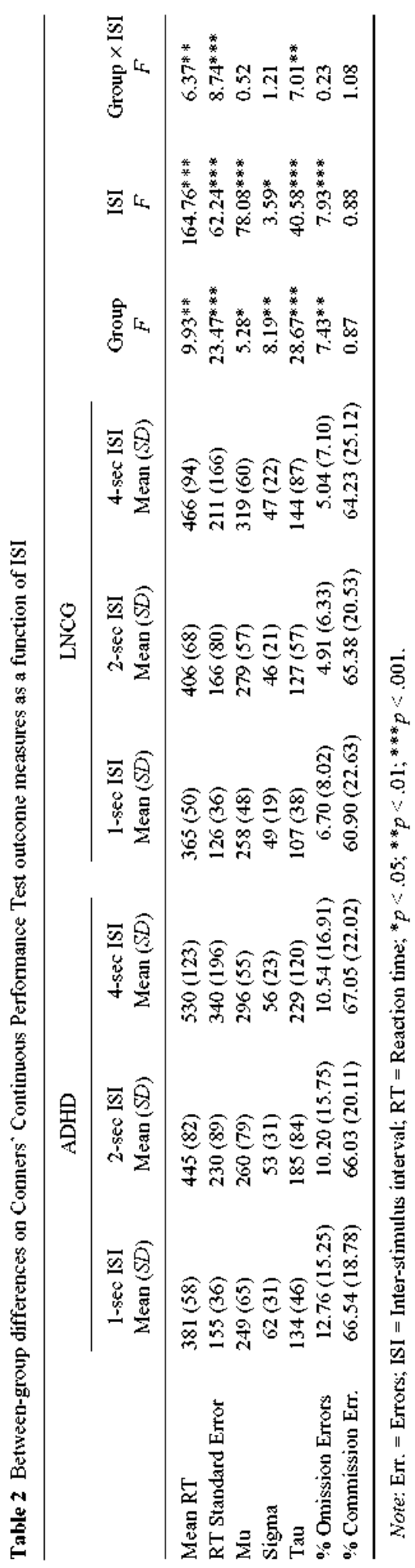

RTSYS interprets any converged value of $\sigma$ or $\tau$ less than mean/100 as indicating a failure and sets all ex-Gaussian parameters as missing. This did not occur for any of the ex-Gaussian parameters for the overall task but did occur for 7 subjects (5 ADHD participants; 2 LNCG participants) when attempting to estimate the ex-Gaussian parameters for individual ISI 
data. These subjects were excluded from analyses using ISI data. However, an additional set of analyses using mean substitution for this missing data confirmed that the pattern of results remained the same if replacement was utilized.

\section{Statistical Analyses}

Performance differences between the ADHD and LNCG group were evaluated using t-tests. First, traditional CPT measures examining errors of omission and errors of commission were used as outcome measures. Next, traditional RT measures (i.e., RT and RT standard deviation) and the RTSYS-produced, ex-Gaussian measures of mu, sigma, and tau were used as outcome measures. Test of equality of variance across groups were performed. If significant, a Satterthwaite correction was used. Finally, in order to examine whether interstimulus interval is related to performance on any of the RT measures, a mixed model ANOVA was conducted using group (ADHD vs. LNCG) and ISI (1, 2, or 4 secs.) as independent variables. Significant interactions of Group x ISI would indicate that differences between groups were affected by ISI.

\section{Results}

\section{Error Pattern Analysis}

No differences were found between the ADHD and LNCG groups for the overall errors of commission $(F(1,128)=0.87, p>.05)$, but a statistically significant difference was found for errors of omission $(F(1,128)=7.43, p<.01)$. Children with ADHD had higher rates of errors of omission than children in the LNCG group.

\section{Reaction Time Analysis}

Traditional RT measures showed that children with ADHD were significantly slower $(F(1,127)=9.93, p<.01)$ and more variable in responding $(F(1,127)=23.47, p<.001)$ than children in the LNCG group. See Table 2. Between-group differences were then compared using the ex-Gaussian measures of RT described above. Using these measures, a different pattern of results emerged. Children with ADHD demonstrated faster reaction times on the mean of the normal component of the ex-Gaussian RT curve, $\mathrm{mu}(F(1,121)=5.28, p<.05)$, and in RT variability in the normal part of the curve, sigma $(F(1,121)=8.19, p<.01)$, compared to normal controls. Finally, the largest difference between groups was shown on the exponential part of the curve, tau $(F(1,121)=28.67, p<.001)$, suggesting that children with ADHD demonstrated much more positive skew in their distribution of RT (see Figure 1).

\section{Performance as a Function of ISI}

Mixed model ANOVAs were conducted using group status (ADHD vs. LNCG) and ISI (1,2, or 4 secs.) as between- and within-subjects variables, respectively. All of the variables evidenced a main effect for ISI with shorter ISIs producing shorter RTs and decreased variance (RT standard error) using traditional RT measures (See Table 2). 


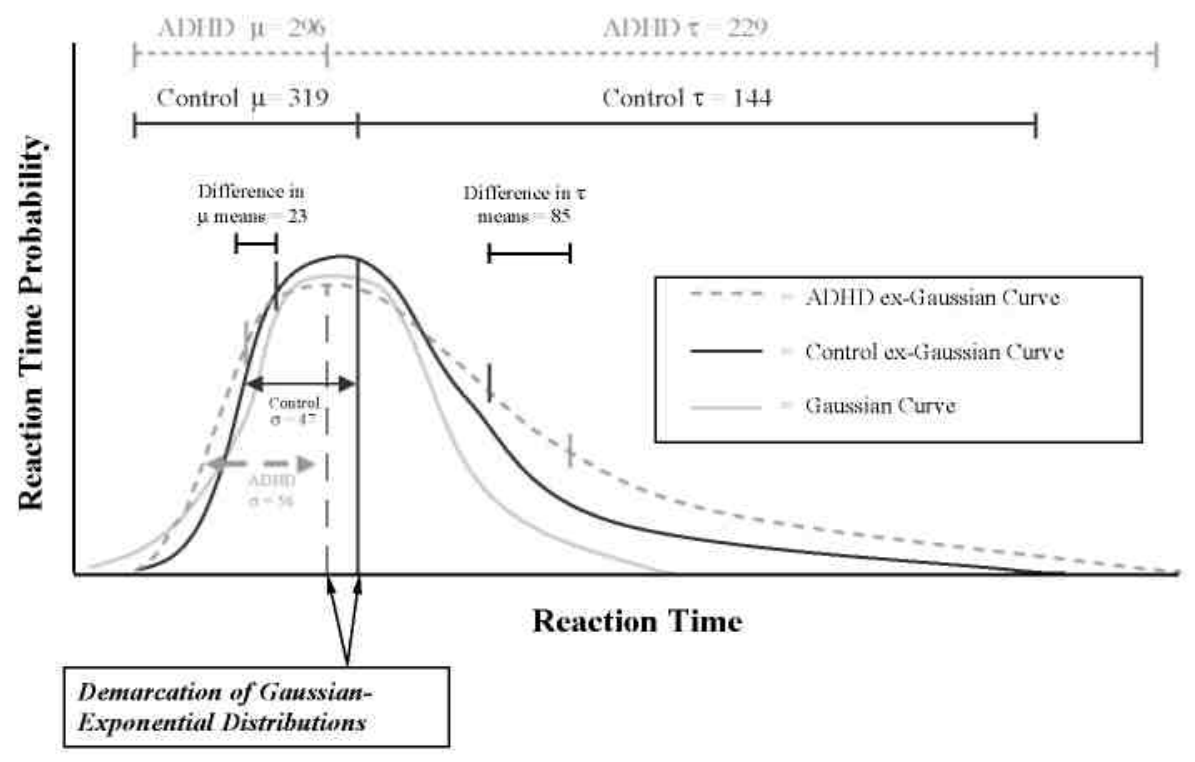

Figure 1 Figure depicting differences in milliseconds between ADHD and LNCG groups on the ex-Gaussian variables mu, sigma, and tau.

On the ex-Gaussian measures of RT, shorter ISIs produced higher values of sigma $(F(2,242)=3.59, p<.05)$ and lower values of $\mathrm{mu}(F(2,242)=78.08, p<.001)$ and tau $(F(2,242)$ $=40.58, p<.001)$. Interactions between group and ISI were observed for mean $\operatorname{RT}(F(2,254)=$ $6.37, p<.01)$, RT standard error $(F(2,254)=8.74, p<.001)$, and tau $(F(2,242)=7.01, p<.01)$. No interaction effect was present for mu or sigma (both $p$ 's $>.05$ ). In general, all three of these interaction effects were present because ISI had little effect on these performance measures within the LNCG group, but they had a profound effect on performance within the ADHD group, revealing greater mean RT, RT standard error, and tau. See Figure 2 for graphs of performance across varying ISIs.

\section{Discussion}

The overall analyses replicate the common finding that children with ADHD responded more slowly and more variably than normal controls matched on gender, age, ethnicity, and collection site as determined by the mean RT and RTSE from the Gaussian distribution. Based on this information alone, the data suggest that the RTs for children with ADHD are slower and more variable than those of matched comparison youth. However, this information assumes that the distribution of these RTs is equal on either side of the mean, leading to the assumption that RTs are simply slower and more variable overall for children with ADHD.

Reaction time distributions were decomposed into two separate theoretical distributions, a Gaussian distribution and an ex-Gaussian distribution, the latter of which specifically accounts for positive skew within the overall data. The introduction of the ex-Gaussian variables, mu, sigma and tau, provided the opportunity to isolate more specifically the variability associated with RT. 


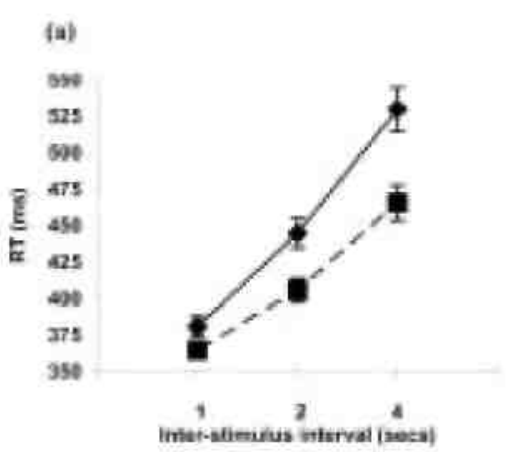

(c)
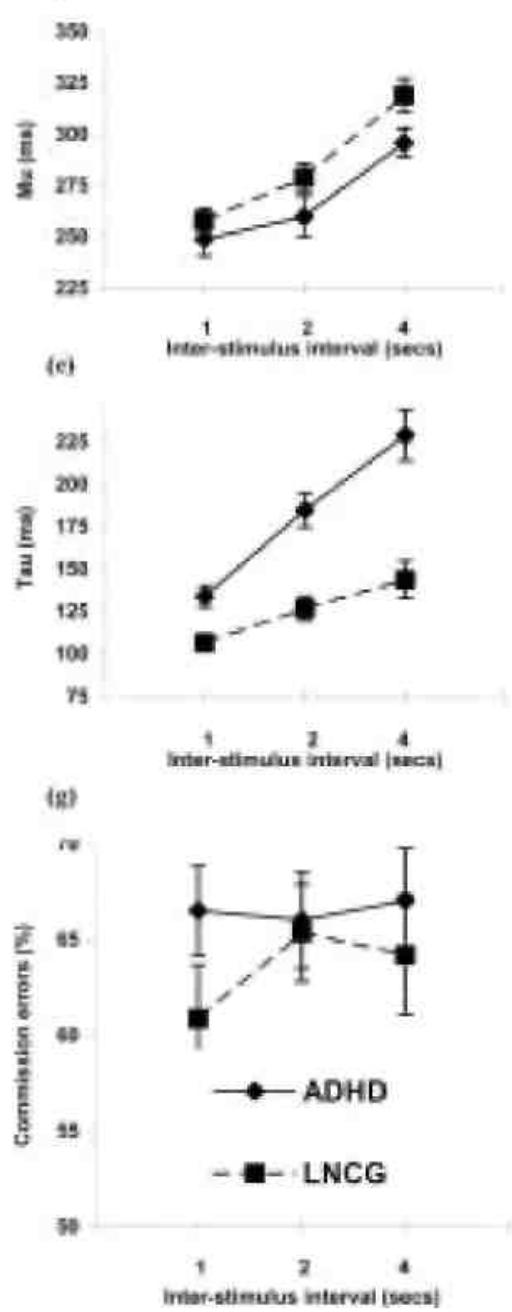
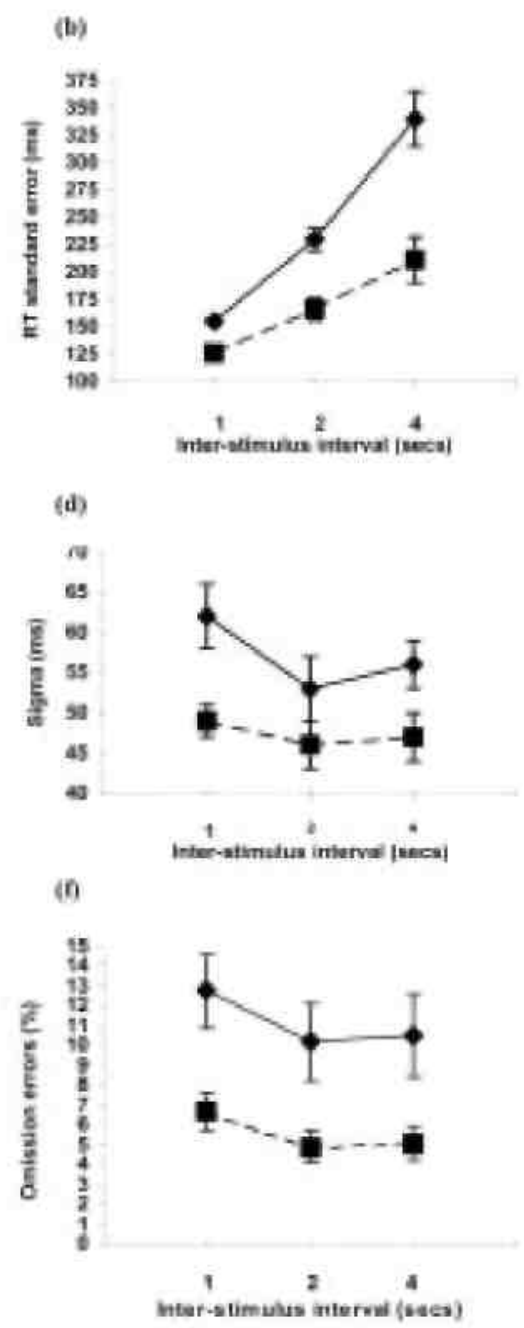

Figure 2 Means (with standard error bars) plotted according to 1-, 2-, and 4-sec interstimulus interval conditions for the Attention Deficit / Hyperactivity Disorder and Local Normal Comparison Groups for a) mean reaction time; b) reaction time standard error; c) mu; d) sigma; e) tau; f) errors of omission; and g) errors of commission on a Go/No-Go task.

The results indicate that children with ADHD were slower in their overall responses associated with the positively skewed portion of the ex-Gaussian curve (tau). That is, children with ADHD had a greater number of RTs that were well beyond their mean performance than did the control group. They also demonstrated no overall difference in the standard deviation of the normal 
portion of the RT distribution curve (sigma). Taking these two findings together, the data indicate that a particular portion of the variability associated with RT performance for children with ADHD differentiates them from normal control children. The ex-Gaussian curve is a more accurate shape for fitting RTs (Luce, 1986). The RT variability that is typically assumed to be evenly distributed across a Gaussian curve for both control children and children with ADHD is actually largely attributable to a particular portion of the RT distribution that is estimated by the exponential component. Children with ADHD differ from the control group primarily in this portion of the variability. Graphically, this difference is represented as a longer tail in the positive direction on a probability density function of RT distribution (see Figure 1).

It is also important to recognize the meaning and implications of a significantly smaller mu for children with ADHD compared to the control group. This finding indicates that children with ADHD demonstrated faster overall mean RT associated with the normally distributed portion of the ex-Gaussian distribution. Children with ADHD are, at times, responding more quickly than controls. This has been seen in previous studies using RT tasks (Tripp \& Alsop, 2001; Douglas, 1999; Koschack et al., 2003). One explanation for this result is that children with ADHD are impulsive responders, though between-group differences were not observed on errors of commission (see discussion below). It should also be noted that mu and tau are typically somewhat negatively correlated, and large values of tau have the potential to deflate values of mu in the process of fitting the ex-Gaussian curve.

It appears that studies identifying slower overall RT as characterized by Gaussian curves are capturing what may be a result of greater variability. In a sense, the exceptionally long RTs that represent greater positive skew may be considered outlier trials that distort the estimate of mean RT. Thus, to characterize overall RT performance as simply slower would be to ignore a pattern of responding that occurs within a block of responses. The analysis of trial-by-trial variability provides a theoretical explanation of moment-by-moment processes that underlie task performance.

The present study findings complement and confirm the results of Leth-Steensen et al. (2000) in terms of finding that the ex-Gaussian tau measure produces the largest differences between ADHD and normal groups. It is notable that these differences were found using quite different tasks. Dissimilar from the Go/No-Go task used in the present study, Leth-Steensen et al. used a discrimination task where subjects saw four empty circles in a line and then one circle was filled in. Subjects were required to press one of four keys, which corresponded to the filled in circle. Surprisingly, while both studies found that children with ADHD have slower mean RT than normal controls, the two studies produced opposite results regarding mu. The present study found that children with ADHD had significantly faster RT than controls associated with the normal portion of the distribution Leth-Steensen et al. found the opposite and statistically significant pattern in their study using the discrimination task, though they did not find that ADHD children were slower at the leading edge of the curve, suggesting that ADHD boys are capable of responding just as quickly as controls. Given the similar sample characteristics across studies, it is reasonable to attribute this inconsistency in findings across studies to differences in the tasks. Indeed, the discrimination task seemed to produce slower overall response times compared to a Go/No-Go task, as RTs were much longer in the Leth-Steensen study. Also, the Go/No-Go task with no warning cues and rapid presentation of stimuli clearly primes an impulsive response style. Together, these may explain why these two tasks would produce opposite patterns of between-group results on this mu variable across studies. However, future research should attempt to specifically investigate these differences in mu with respect to the aforementioned 
differences in test demands (i.e., target rate, warning cues).

\section{Interstimulus Interval Effects}

To better understand the group differences, performance across the three ISIs were analyzed. Reaction time performance is known to vary directly with ISI in normal subjects (Niemi \& Näätänen, 1981). ISI may exert its effect by providing more opportunity for distraction or off-task behavior, or by allowing less time to measure RT, or possibly by changing the cognitive energy level (Sergeant \& van der Meere, 1994) of the subject. These factors may differentially improve or impair the performance of children with ADHD. The present study found that RT and RT variability increased as ISI increased. Further, the ex-Gaussian parameters mu and tau increased as ISI increased. In summary, as children were given less time between trials, RT became faster and performance became less variable, establishing a differential temporal gradient between children with ADHD and controls. One explanation for this pattern of results may be that the shorter ISI conditions ( 1 and $2 \mathrm{sec}$ ) primed an impulsive response style. Another explanation is that the shorter ISI did not allow for extreme values of positive skew because the succeeding trial cut short the responding period, therefore truncating the RT distribution. Either set of circumstances, nevertheless, highlights the importance of temporal variation in the RT performance of children with ADHD.

\section{Theoretical Implications}

How does the present pattern of findings contribute to theoretical models of ADHD? Higher RT variability in the ADHD group compared to normal controls is consistent with but not specific to most broadly based theoretical perspectives. In his model of behavioral inhibition, Barkley (Barkley, 1997b) places a strong emphasis on temporal estimation. He predicts that the "insertion of temporal delays into. . . tasks should more adversely affect the performance of those with ADHD than control groups, with longer durations associated with a correspondingly greater likelihood that such group differences will emerge" (Barkley, 1997a, p. 274). Though a rather general prediction, this is consistent with the results from the present study, particularly across interstimulus intervals.

Douglas has provided another theoretical model of ADHD. She has suggested that cognitive impairments associated with ADHD are better viewed as a self-regulatory defect, or a failure to allocate adequate effort to meet task demands (Douglas, 1999). For more than 30 years she has presented and supported the idea that attentional functioning is impaired in ADHD, though she argues that response inhibition is a fundamental characteristic differentiating children with ADHD, as well. Douglas highlights attention dysregulation, emphasizing that a broad pattern of variability in performance across a wide range of tasks is a reflection of this dysregulation in ADHD. The present results support this notion and contribute to a body of work documenting this variability. Moreover, these results replicate findings from previous work (Leth-Steensen, Elbaz, \& Douglas, 2000) and provide further evidence for performance variability as fundamental to ADHD.

Finally, Sergeant, Oosterlaan, and van der Meere (1999) present a cognitive-energetic model of ADHD. Consistent with previous models, it posits that inhibitory control and self-regulation are central to this disorder. However, they add an energetic component to the model, which posits that an optimal energetic level must be present in order for a person to respond to a stimulus. According to their theory, the ability to inhibit responses is dependent on the subject's ability to put forth effort suitable to generate adequate arousal and activation in order to process information. They emphasize that variable motor output is an expression of attentional impairment, and that this impairment results from the same neurological dysfunction that gives rise to poor response inhibition, which is the primary underlying deficit in ADHD (Sergeant, 2000; Sergeant \& van der Meere, 1994). Hence, by virtue of poor motor output, 
children with ADHD should demonstrate more variability in RT. Furthermore, as suggested by Sergeant et al. (1999), if the basis of poor performance on tests of cognitive functioning has to do with motor output, a significant part of which is motor preparation, then more time provided for motor preparation could result in greater opportunity for error. The present study showed that RT speed and variability worsened with longer ISIs consistent with this idea.

Whereas theoretical underpinnings are consistent with the present study findings in regards to RT variability, neither theory nor most previous research would have predicted the lack of group differences in errors of commission. All theories of ADHD suggest that response inhibition is impaired. Most empirical investigations using a variety of neuropsychological tasks have confirmed this phenomenon. In a meta-analytic study, the effect size for errors of commission on CPT tasks was .73 (Losier, McGrath, \& Klein, 1996). Several authors have suggested that Go/No-Go tasks, particularly the Conners' CPT, establish a response bias that highlights impulsive responding and poor response inhibition more than other traditional CPTs (Ballard, 2001; Epstein, Johnson, Varia, \& Conners, 2001). Indeed, group differences on errors of commission were observed in an epidemiologically derived sample using the same Go/No-Go task used in this study (Conners, Epstein, Angold, \& Klaric, 2003). Thus, it is perplexing that no group differences were observed on errors of commission in the present study. The study was sufficiently powered, contained well-defined groups and was able to find differences on other outcome measures consistent with the literature.

\section{Limitations and Future Directions}

There are several limitations in the present study that need to be considered when interpreting the findings. First, the matched sample that was used in this study did not match for psychiatric comorbid conditions. It is possible that part of the performance differences between groups may be a result of differences in comorbidities across groups and not solely ADHD. Our sample selection process for the present study also excluded the majority of the MTA sample because of their not meeting diagnostic criteria at 24 months and/or their current receipt of medications for ADHD. Given the question this study sought to answer, excluding these subjects was necessary. Medication will undoubtedly affect performance on this task. A separate publication discusses these medication-related results (see Epstein, Conners, Hervey, et al., in press). Including children with a positive ADHD diagnosis but currently taking medication for ADHD in our ADHD group would have confounded our sample. Thus, a decision was made to include patients as long as they were off medication for the day of the testing. Some of our patients in the ADHD group may have had a medication history as recent as the previous day.

The findings from the present study point to clear differences in RT performance between controls and children with ADHD. However, they also leave several questions unanswered and raise new ones with regard to both specific aspects of the present study design and general issues within the field at large. First, specific issues related to study design that should be addressed in future research have to do with the organization and order of RT trials and blocks of trials. The findings in the present study suggest that performance varies across ISI. Therefore, varying the number of trials per ISI block, or simply varying the ISI block beyond 1-, 2-, and 4-seconds in duration may change response patterns. In addition, all blocks included exactly 20 trials. The influence of the distribution of blocks of trials on performance is unclear. It may be that blocks of a smaller, larger, or frequently varied number of trials could influence performance by changing the trial expectancy that likely develops across blocks as the test progresses. In addition, the overall length of the testing period may also influence performance, depending upon whether individuals with ADHD tend to demonstrate a relative decrease in performance 
across time, or if they simply perform poorly from the outset. An additional aspect to consider has to do with the medium in which the stimuli were presented. Neuropsychology has historically differentiated performance based on stimulus type. The present study used the Conners' CPT, which included a visual presentation. However, RT data can be readily collected using similar tests assessing performance based on auditory stimuli, which may reveal different performance patterns.

From a larger more general perspective, the findings in the present study do not compare pure ADHD subtypes to one another due to inadequate sample size. It is unknown whether subtype performance might vary. Certainly ADHD subtype has had some bearing on performance on cognitive tests in previous research. A theoretical argument has been made that RT performance and greater tau is primarily influenced by inattention (Leth-Steensen, Elbaz, \& Douglas, 2000). It would therefore be theoretically important to determine if the effects found in the present study are primarily driven by symptoms of inattention. This would also provide the opportunity for direct theoretical testing, something that several authors have suggested is highly important for advancements in the study of ADHD (Castellanos \& Tannock, 2002; Sergeant, 2000).

\section{Acknowledgements}

The MTA is a cooperative treatment study performed by six independent research teams in collaboration with the staff of the Division of Clinical and Treatment Research of the National Institute of Mental Health (NIMH), Rockville, Maryland and the Office of Special Education Programs (OSEP) of the U.S. Department of Education (DOE). The NIMH Principal Collaborators are Peter S. Jensen, M.D., L. Eugene Arnold, M.Ed., M.D., John E. Richters, Ph.D., Joanne B. Severe, M.S., Donald Vereen, M.D., and Benedetto Vitiello, M.D. Principal Investigators and coinvestigators from the six sites are as follows: University of California at Berkeley/San Francisco (UO1 MH50461): Stephen P. Hinshaw, Ph.D., Glen R. Elliott, M.D., Ph.D.; Duke University (UO1 MH50447): C. Keith Conners, Ph.D., Karen C. Wells, Ph.D., John S. March, M.D., M.P.H.; University of California at Irvine/Los Angeles (UO1 MH50440): James M. Swanson, Ph.D.; Dennis P. Cantwell, M.D.; Timothy Wigal, Ph.D.; Long Island Jewish Medical Center/Montreal Children's Hospital (UO1 MH50453): Howard B. Abikoff, Ph.D., Lily Hechtman, M.D.; New York State Psychiatric Institute/Columbia University/Mount Sinai Medical Center (UO1 MH50454): Laurence L. Greenhill, M.D., Jeffrey H. Newcorn, M.D.; University of Pittsburgh (UO1 MH50467): William E. Pelham, Ph.D., Betsy Hoza, Ph.D. Helena C. Kraemer, Ph.D. (Stanford University) is statistical and design consultant. The OSEP/DOE Principal Collaborator is Thomas Hanley, Ed.D. Aaron Hervey, Ph.D. was supported on this project by a midcareer development award to the second author (K24 MH64478).

\section{References}

American Psychiatric Association. (1994). Diagnostic and statistical manual of mental disorders (4th ed.). Washington, DC: Author.

Arnold, L. E., Abikoff, H. B., Cantwell, D. P., Conners, C. K., Elliott, G., Greenhill, L. L., Hechtman, L., Hinshaw, S. P., Hoza, B., Jensen, P. S., Kraemer, H. C., March, J. S., Newcorn, J. H., Pelham, W. E., Richters, J. E., Schiller, E., Severe, J. B., Swanson, J. M., Vereen, D., \& Wells, K. C. (1997). NIMH Collaborative Multimodal Treatment Study of Children with ADHD (the MTA). Design challenges and choices. Archives of General Psychiatry, 54(9), 865-870.

Ballard, J. C. (2001). Assessing attention: Comparison of response-inhibition and traditional continuous performance tests. Journal of Clinical and Experimental Neuropsychology, 23(3), 331-350.

Barkley, R. A. (1997a). ADHD and the nature of self-control. New York: Guilford Press.

Barkley, R. A. (1997b). Behavioral inhibition, sustained attention, and executive functions: Constructing a unifying theory of ADHD. Psychological Bulletin, 121, 65-94.

Burbeck, S. L., \& Luce, R. D. (1982). Evidence from auditory simple reaction times for both change and level detectors. Perception \& Psychophysics, 32, 117-133.

Castellanos, X., \& Tannock, R. (2002). Neuroscience of attention-deficit/hyperactivity disorder: The search for endophenotypes. Nature Reviews Neuroscience, 3, 617-628. 
Cohen, N. J., \& Douglas, V. I. (1972). Characteristics of the orienting response in hyperactive and normal children. Psychophysiology, 9, 238-245.

Conners, C. K. (1994). Conners' continuous performance test computer program, version 3.0. North Tonawanda, NY: Multi-Health Systems, Inc.

Conners, C. K., Epstein, J. N., Angold, A., \& Klaric, J. (2003). Continuous performance test performance in a normative epidemiological sample. Journal of Abnormal Child Psychology, 31, 555-562.

Denney, C. B., \& Rapport, M. D. (2001). Cognitive pharmacology of stimulants in children with ADHD. In M.V. Solanto, A. F. T. Arnsten, \& F. X. Castellanos (Eds.), Stimulant drugs and ADHD: Basic and clinical neuroscience (pp. 283-302). New York: Oxford University Press.

Douglas, V. I. (1999). Cognitive control processes in attention-deficit/hyperactivity disorder. In H. C. Quay, \& A. E. Hogan (Eds.), Handbook of disruptive behavior disorders (pp. 105-138). New York: Kluwer Academic/Plenum Publishers.

Epstein, J. N., Conners, C. K., Hervey, A. S., Tonev, S. T., Arnold, L. E., Abikoff, H. B., Elliott, G., Greenhill, L. L., Hechtman, L., Hoagwood, K., Hinshaw, S. P., Hoza, B., Jensen, P. S., Kraemer, H. C., March, J. S., Newcorn, J. H., Pelham, W. E., Severe, J. B., Swanson, J. M., Wells, K., Vitiello, B., \& Wigal, T. of the MTA Cooperative Study Group. (in press). Neuropsychological outcomes in the Multimodal Treatment Study of ADHD (MTA) at 10-months post-treatment. Journal of Child Psychology \& Psychiatry.

Epstein, J. N., Johnson, D. E., Varia, I. M., \& Conners, C. K. (2001). Nuropsychological assessment of response inhibition in adults with ADHD. Journal of Clinical and Experimental Neuropsychology, 23, 362-371.

Giedd, J. N., Blumenthal, J., Molloy, E., \& Castellanos, F. X. (2001). Brain imaging of attention deficit/hyperactivity disorder. Annals of the New York Academy of Sciences, 931, 33-49.

Heathcote, A. (1996). RTSYS: A DOS application for the analysis of reaction time data. Behavior Research Methods, Instruments, \& Computers, 28, 427-445.

Hervey, A. S., Epstein, J. N., \& Curry, J. F. (2004). The neuropsychology of adults with ADHD: A meta-analytic review. Neuropsychology, 18(3), 485-503.

Howell, D. C. (1989). Fundamental statistics for the behavioral sciences (2nd ed.). Boston, MA: PWS-Kent.

Keppel, G. (1991). Design and analysis: A researcher's handbook (3rd ed.). Upper Saddle River, NJ: Prentice-Hall, Inc.

Iaboni, F., Douglas, V. I., \& Ditto, B. (1997). Psychophysiological response of ADHD to reward and extinction. Psychophysiology, 34(1), 116-123.

Koschack, J., Kunert, H. J., Derichs, G., Weniger, G., \& Irle, E. (2003). Impaired and enhanced attentional function in children with attention deficit/hyperactivity disorder. Psychological Medicine, 33, 481-489.

Klorman, R., Salzman, L. F., Pass, H. L., Borgstedt, A. D., \& Dainer, K. B. (1979). Effects of methylphenidate on hyperactive children's evoked responses during passive and active attention. Psychophysiology, 16, 23-29.

Kuntsi, J., Oosterlaan, J., \& Stevenson, J. (2001). Psychological mechanisms in hyperactivity. I. Response inhibition deficit, working memory impairment, delay aversion, or something else? Journal of Child Psychology and Psychiatry, 42(2), 199-210.

Kuntsi, J., \& Stevenson, J. (2001). Psychological mechanisms in hyperactivity: II The role of genetic factors. Journal of Child Psychology and Psychiatry, 42(2), 211-219.

Leth-Steensen, C., Elbaz, Z. K., \& Douglas, V. I. (2000). Mean response times, variability, and skew in the responding of ADHD children: A response time distributional approach. Acta Psychologica, 104, 167-190.

Losier, B. J., McGrath, P. J., \& Klein, R. M. (1996). Error patterns on the continuous performance test in non-medicated and medicated samples of children with and without ADHD: A meta-analytic review. Journal of Child Psychology and Psychiatry, 37, 97-987.

Luce, R. D. (1986). Response times: Their role in inferring elementary mental organization. New York: Oxford University Press.

MTA Coopertive Group. (1999). A 14-month randomized clinical trial of treatment strategies for attention-deficit/hyperactivity disorder. Archives of General Psychiatry, 56, 1073-1086.

Niemi, P., \& Näätänen, R. (1981). Foreperiod and simple reaction time. Psychological Bulletin, 89, 133-162.

Pennington, B., Groisser, L., \& Welsh, R. (1993). Contrasting cognition deficits in ADHD versus reading disabilities. Developmental Psychology, 29, 511-523.

Pennington, B., \& Ozonoff, S. (1996). Executive functions and developmental psychopathology. Journal of Child Psychology and Psychiatry, 37, 51-87. 
Ridderinkhof, K. R. (2002). Activation and suppression in conflict tasks: Empirical clarification through distributional analyses. In W. Prinz, \& B. Hommel (Eds.), Common mechanisms in perception and action. Attention \& performance, (Vol. XIX, pp. 494-519). Oxford: Oxford University Press.

Ridderinkhof, K. R., Scheres, A., Oosterlaan, J., \& Sergeant, J. A. (in press). Distribution-analytical techniques in the study of AD/HD: Delta plot analyses reveal deficits in response inhibition that are eliminated by methylphenidate treatment. Journal of Abnormal Psychology.

Sergeant, J. (2000). The cognitive-energetic model: an empirical approach to attention-deficit hyperactivity disorder. Neuroscience and Biobehavioral Reviews, 24, 7-12.

Sergeant, J., \& van der Meere, J. (1990). Convergence of approaches in localizing the hyperactivity deficit. In B. B. Lahey, \& A. E. Kazdin (Eds.), Advances in clinical child psychology, (Vol. 13, pp. 207-245). New York: Plenum.

Sergeant, J., \& van der Meere, J. (1994). Toward an empirical child psychopathology. In D. K. Routh (Ed.), Disruptive behavior disorders in childhood (pp. 59-85). New York: Plenum.

Sergeant, J., Oosterlaan, J., \& van der Meere, J. (1999). Information processing and energetic factors in attention-deficit/hyperactivity disorder. In H. C. Quay, \& A. E. Hogan (Eds.), Handbook of disruptive behavior disorders (pp. 75-104). New York: Kluwer Academic/Plenum Publishers.

Shaffer, D., Fisher, P., Dulcan, M. K., Davies, M., Piacentini, J., Schwabstone, M. E., Lahey, B. B., Bourdon, K., Jensen, P. S., Bird, H. R., Canino, G., \& Regier, D. A. (1996). The NIMH diagnostic interview schedule for children version 2.3 (DISC-2.3): Description, acceptability, prevalence rates, and performance in the MECA study. Methods for the epidemiology of child and adolescent mental disorders study. Journal of the American Academy of Child \& Adolescent Psychiatry, 35(7), 856-877.

Swanson, J. M. (1992). School-based assessments and interventions for ADD students. Irvine, CA: K. C. Publications.

Teicher, M. H., Ito, Y., Glod, C. A., \& Barber, N. I. (1996). Objective measurement of hyperactivity and attentional problems in ADHD. Journal of the American Academy of Child and Adolescent Psychiatry, 35(3), 334-342.

Tripp, G., \& Alsop, B. (2001). Sensitivity to reward delay in children with attention deficit hyperactivity disorder (ADHD). Journal of Child Psychology and Psychiatry, 42, 691-698.

Ulrich, R., \& Miller, J. (1994). Effects of truncation on reaction time analysis. Journal of Experimental Psychology: General, 123, 34-80.

Van der Molen, M. W. (1996). Energetics and the reaction process: Running threads through experimental psychology. In O. Neumann, \& A. F. Sanders (Eds.), Handbook of perception and action, (Vol. III, pp. 229-275). London: Academic Press.

Zahn, T. P., Kruesi, M. J. P., \& Rapoport, J. L. (1991). Reaction time indices of attention deficits in boys with disruptive behavior disorders. Journal of Abnormal Child Psychology, 19, 233-252. 